\title{
Communication Range Dynamics Using an Energy Saving Self-Adaptive Transmission Power Controller in a Wireless Sensor Network
}

Néstor Lucas Martínez ${ }^{1, *}$, José-Fernán Martínez Ortega ${ }^{1, \dagger}$, Vicente Hernández Díaz ${ }^{1, \dagger}$ and Raúl M. del Toro ${ }^{2, \dagger}$

${ }^{1}$ Centro de Investigación en Tecnologías Software y Sistemas Multimedia para la Sostenibilidad (CITSEM), Universidad Politécnica de Madrid, Calle Alan Turing 3, 28031 Madrid, Spain;

E-Mails: jf.martinez@upm.es (J.-F.M.O.); vicente.hernandez@upm.es (V.H.D.)

${ }^{2}$ Centro de Automática y Robótica, Universidad Politécnica de Madrid, Carretera Campo Real Km. 0.2, 28500 Arganda del Rey, Spain; E-Mail: raul.deltoro@ car.upm-csic.es

* Author to whom correspondence should be addressed; E-Mail: nestor.lucas@upm.es;

Tel.: +34-91-452-4900 (ext. 20794); Fax: +34-91-336-7817.

Published: 11 November 2015

\begin{abstract}
The deployment of the nodes in a Wireless Sensors and Actuators Network (WSAN) is typically restricted by the sensing and acting coverage. This implies that the locations of the nodes may be, and usually are, not optimal from the point of view of the radio communication. And also when the transmission power is tuned for those locations, there are other unpredictable factors that can cause connectivity failures, like interferences, signal fading due to passing objects, and of course, radio irregularities. A control based self-adaptive system is a typical solution to improve the energy consumption while keeping a good connectivity. In this paper, we explore how the communication range for each node evolves along the iterations of an energy saving self-adaptive transmission power controller when using different parameter sets in an outdoor scenario, providing a WSAN that automatically adapts to surrounding changes keeping a good connectivity. The results obtained in this paper show how the parameters with the best performance keep a k-connected network, where $\mathrm{k}$ is in the range of the desired node degree plus or minus a specified tolerance value. In addition, the worst performance shows how a bad parameters choice can create isolated islands, groups of nodes disconnected from the rest of the network.
\end{abstract}


Keywords: self-adaptive systems; wireless sensor networks; transmission power control; connectivity; communication range

\section{Introduction}

The Wireless Sensors and Actuators Networks (WSAN) are a well known wireless communication technology with benefits that are becoming significantly important for solving upcoming societies challenges [1]. The WSAN reliability is strongly affected by unpredictable changes in the environment. A node transmitting using always its maximum power, will show a reliability highly immunized to changes in the environment, but the node energy consumption will be unnecessarily soaring. Thus, a trade-off between energy consumption and communication reliability is required, as proposed in diverse strategies (Kotian et al. [2], Mahmood et al. [3] and Kusy et al. [4], among others). In a previous work [5], we propose a self-adaptive strategy, based on fuzzy control, which adapts each node transmission power to achieve an optimal number of neighbors (an optimal node degree). This optimal number of neighbors guarantees the node a high likelihood to reach any other node in the WSAN, and depends on parameters of the specific WSAN: the deployment area and the number of nodes per $\mathrm{m}^{2}$. The node transmission power is dynamically adapted and thus the energy consumption is optimized.

The proposed fuzzy control system running in each node in a WSAN includes two feedback control loops. A primary feedback control manages the node transmission power considering both its real and targeted number of neighbors. A secondary feedback control loop manages the node targeted or optimal number of neighbors considering the battery level. In each feedback control loop there is a decision-making function based on fuzzy logic that actually decides what to do at each moment. The system itself is described in a previous work [6].

In this paper we discuss about the achievements of a real system accomplishing the approach above described, focusing on each node performance. Global WSAN figures were also discussed in [6].

\section{Experimental Set-Up}

Ten experiments were conducted using eight SunSPOT nodes following the distribution described in Figure 1. The orientation of the antenna was arbitrary.

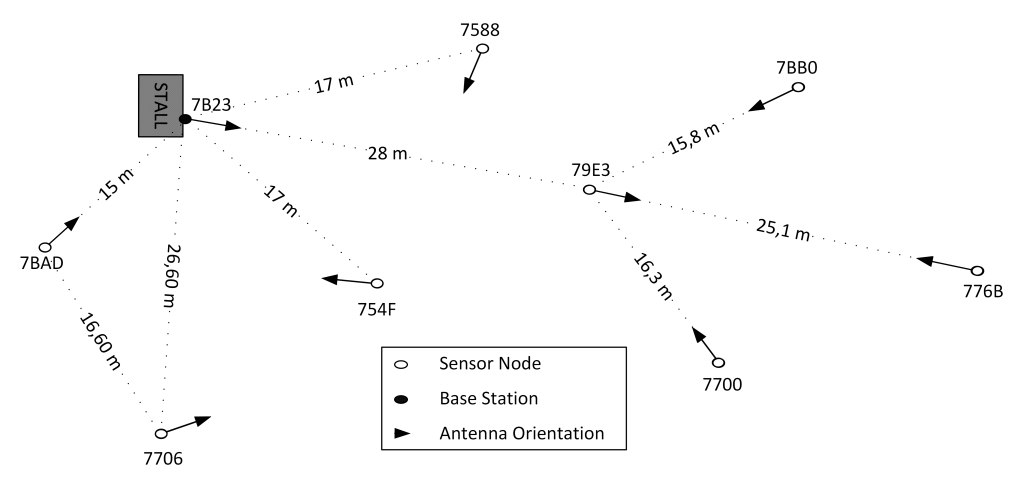

Figure 1. Deployment, distances and nodes orientation. 
The first two experiments, $\mathrm{e} 01$ and $\mathrm{e} 02$, were control experiments using a fixed transmission power instead of the self-adaptive system. Experiments e03 to e10 used different configuration parameters for the self-adaptive system as shown in Table 1 . The parameters were the reference or targeted node degree $\left(N D_{R}\right)$, the tolerance over the reference node degree $\left(\xi_{N D}\right)$, the transmission power change rate scale $\left(k_{C R}\right)$ and the energy critical level $\left(E_{C R}\right)$, a threshold to reduce the energy consumption even more.

Table 1. Parameters and results from previous work.

\begin{tabular}{c|cccc|cc}
\hline Experiment & $\begin{array}{c}\boldsymbol{N D}_{\boldsymbol{R}} \\
\text { Reference ND }\end{array}$ & $\begin{array}{c}\xi_{\boldsymbol{N D}} \\
\text { Tolerance on } N D_{R}\end{array}$ & $\begin{array}{c}\boldsymbol{k}_{\boldsymbol{C} \boldsymbol{R}} \\
\text { CR change rate }\end{array}$ & $\begin{array}{c}\overline{\boldsymbol{E}_{\boldsymbol{C} \boldsymbol{R}}} \\
\text { Critical energy level }\end{array}$ & $\begin{array}{c}\boldsymbol{J}_{\boldsymbol{e}} \\
(\mathrm{mAh})\end{array}$ & $\boldsymbol{J}_{\boldsymbol{c}}$ \\
\hline $\mathrm{e} 03$ & 2 & 0 & 1 & 150 & 3921.53 & 36.7036 \\
$\mathrm{e} 04$ & 2 & 0 & 3 & 150 & 3947.76 & 17.0662 \\
$\mathrm{e} 05$ & 2 & 1 & 3 & 150 & 3639.89 & 9.6857 \\
$\mathrm{e} 06$ & 3 & 1 & 3 & 150 & 3817.46 & 12.9013 \\
$\mathrm{e} 07$ & 3 & 0 & 3 & 150 & 3846.54 & 11.3045 \\
$\mathrm{e} 08$ & 3 & 0 & 1 & 150 & 3865.22 & 19.0762 \\
$\mathrm{e} 09$ & 3 & 1 & 1 & 150 & 3798.83 & 20.1254 \\
$\mathrm{e} 10$ & 2 & 1 & 1 & 150 & 3952.63 & 40.1113 \\
\hline
\end{tabular}

The previously obtained values for the cost of the global energy consumption $\left(J_{e}\right)$ and the global connectivity $\left(J_{c}\right)$ are also shown in Table 1 .

\section{Results and Discussion}

In Table 1 we can see that the best (lowest) values were obtained in experiment e05, while the worst (highest) are those from experiment e10. The worst scenario was due to the configuration parameters allowing a node to reach a steady state with just one neighbor. Of course this implies the creation of isolated islands. But this also happens with other configurations.

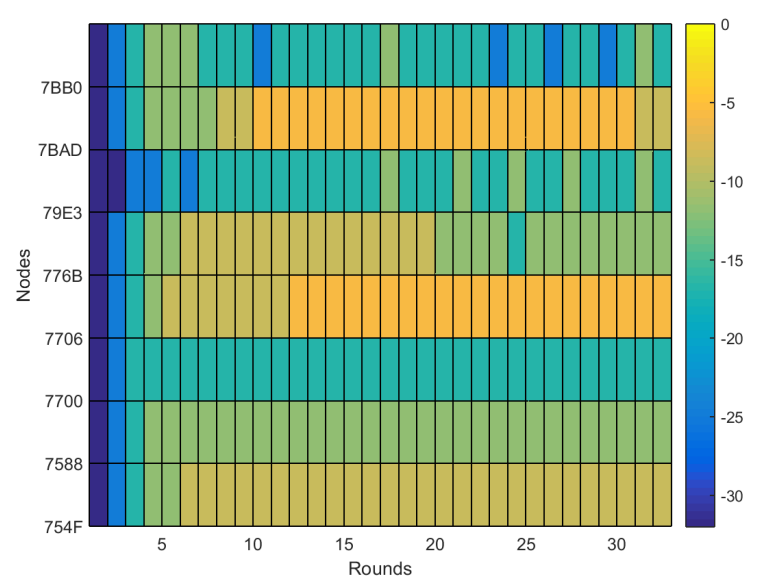

(a)

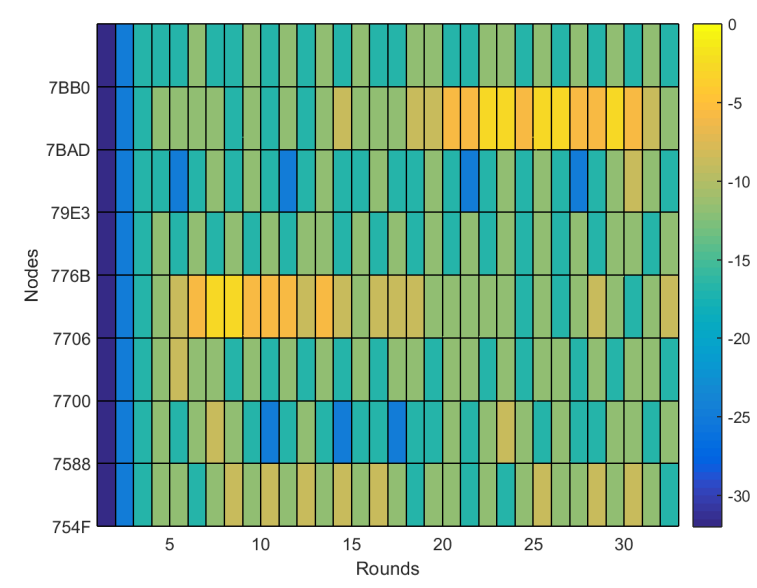

(b)

Figure 2. $P_{T X}$ dynamics comparison; (a) $P_{T X}$ dynamics for experiment e05; (b) $P_{T X}$ dynamics for experiment e10. 
For instance the next worst scenario regarding the cost due to energy consumption $\left(J_{e}\right)$ was e04. In Figure $2 \mathrm{a}$ and Figure $2 \mathrm{~b}$ we can see the communication range dynamics for experiments e05 and e04 respectively. Each color is assigned to a transmission power. Therefore each color change represents a change in the transmission power.

In Figure $3 \mathrm{a}$ and Figure $3 \mathrm{~b}$ we can see the evolution of the number of neighbors for both experiments $\mathrm{e} 05$ and $\mathrm{e} 04$. As can be observed even in the best case, that is, in experiment e05, there are nodes that from occasionally lose all their neighbors. This situation is worse in experiment e04.

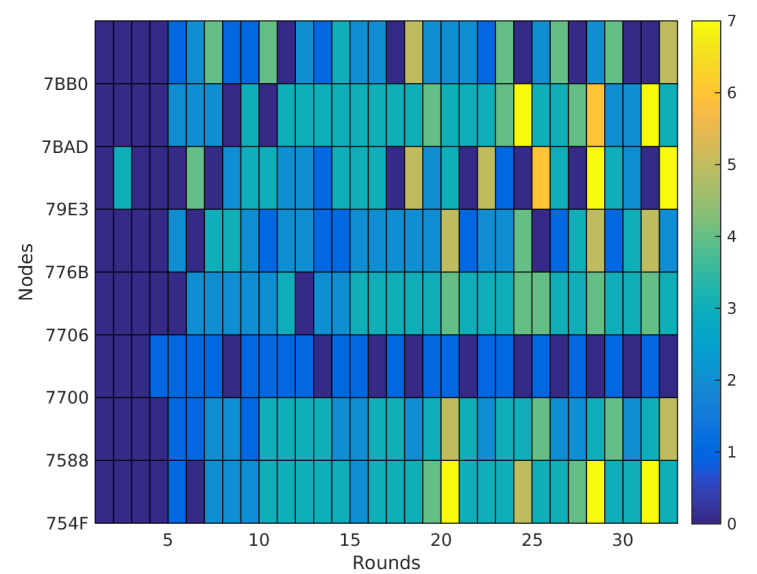

(a)

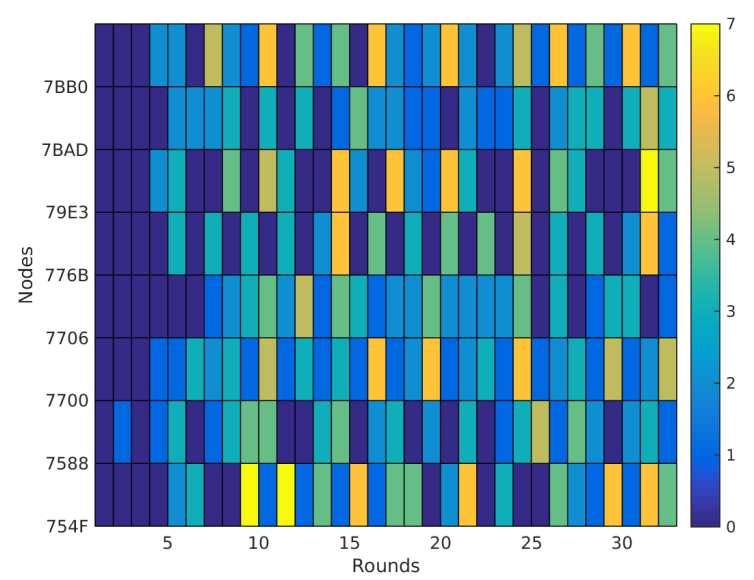

(b)

Figure 3. $N D$ dynamics comparison; (a) $N D$ dynamics for experiment e05; (b) $N D$ dynamics for experiment e10.

Equation 1 is then used to calculate the global cost of the communication range dynamics introduced by the control based self-adaptive system parameters. In the equation $J_{d}$ refers to the cost due to the communication range dynamics, while $\chi_{i}$ refers to the transmission power changes done in node $i$, and $n$ is the total number of nodes used in the experiment. In this case we have considered the same amount of rounds for every experiment, so we can compare the results.

$$
J_{d}=\sum_{i=0}^{n} \chi_{i}
$$

Applying Equation 1 to the whole set of experiments using the self-adaptive system we obtain the results shown in Table 2 .

As we can observe from the results, there is a direct relation between the usage of a non-zero $\xi_{N D}$ value and the number of changes observed in the transmission power during each experiment. We have obtained that, for the experiments where $\xi_{N D}>0$, the average of changes $\mu_{\xi_{N D}>0}$ is 88 , with a standard deviation $\sigma_{\xi_{N D}>0}$ is 28.6473. Similarly for the experiments where $\xi_{N D}=0$ the average of changes $\mu_{\xi_{N D}=0}$ is 187 , with a standard deviation $\sigma_{\xi_{N D}=0}$ is 27.6526 . And again the best results are obtained in experiment e05. 
Table 2. Cost of the communication range dynamics and related configuration parameters.

\begin{tabular}{c|cccc|c}
\hline Experiment & $\boldsymbol{N D}_{\boldsymbol{R}}$ & $\boldsymbol{\xi}_{\boldsymbol{N} \boldsymbol{D}}$ & $\boldsymbol{k}_{\boldsymbol{C R}}$ & $\boldsymbol{E}_{\boldsymbol{C R}}$ & $\boldsymbol{J}_{\boldsymbol{d}}$ \\
\hline $\mathrm{e} 03$ & 2 & 0 & 1 & 150 & 150 \\
$\mathrm{e} 04$ & 2 & 0 & 3 & 150 & 211 \\
$\mathrm{e} 05$ & 2 & 1 & 3 & 150 & 58 \\
$\mathrm{e} 06$ & 3 & 1 & 3 & 150 & 83 \\
$\mathrm{e} 07$ & 3 & 0 & 3 & 150 & 205 \\
$\mathrm{e} 08$ & 3 & 0 & 1 & 150 & 182 \\
$\mathrm{e} 09$ & 3 & 1 & 1 & 150 & 127 \\
$\mathrm{e} 10$ & 2 & 1 & 1 & 150 & 84 \\
\hline
\end{tabular}

\section{Conclusions and Outlook}

As we can observe from the results, it seems that there is some time at which there is a communication range adjustment, even when the network have reached a previous steady state. This implies that even using the same transmission power in the same node for the same deployment and conditions, the communication range varies over time. The variability of the communication range when using the same transmission power can be caused by interferences in the area. But there is also another interesting phenomena. In our implementation we have used a basic neighbor discovery protocol that considers that two nodes are neighbors if they can communicate each other in just one hop.

We have used directly the communication capabilities of the radio system, which has a receiver sensitiby of $-95 \mathrm{dBm}$. IEEE 802.15.4 requires a 1\% PER (Packet Error Rate) at $-85 \mathrm{dBm}$. This means that our nodes can receive packets with as low as -95 dBM RSSI (Receiver Strength Signal Indicator), but of course, with higher PER. Therefore there can be neighboring nodes with higher PER causing that, from one round to the next one, they cannot see each other. Thus they require a transmission power adjustment, which can require a new readjustmen in successive rounds. One simple solution for this issue can be using a filter function in the neighboring decision, so that any potential neighbor is considered as such only if it complies with certain quality parameters, like a good RSSI or a good LQI (Link Quality Indication). Another potential solution considered for further exploration can be using a fuzzy decision making method so instead of being a binary relationship among the nodes (being neighbor or not), it can be a probabilistic one, assigning a weight between 0 and 1 to each possible neighbor.

Finally, another worth thing for further research is the self-adjusting of the reference node degree and communication range change rate depending on the density of nodes in the area. After all, this density depends on the sensing coverage, and the communication range to assure certain cinnectivity is directly related to it [7].

\section{Acknowledgments}

This work has been supported by the European project "Design, Monitoring, and Operation of Adaptive Networked Embedded Systems" (DEMANES). It has been funded by ARTEMIS-JU (projects code ARTEMIS-JU 295372) and "Ministerio de Industria, Energía y Turismo" of Spain (project code ART-010000-2012-002). 


\section{Author Contributions}

Raúl M. del Toro has focused on designing the self-adaptive strategy by using control engineering methods and on defining the corresponding fuzzy-logic functions. Vicente Hernández Díaz, José-Fernán Martínez and Néstor Lucas Martínez have analyzed and studied the suitability and feasiblity of the tested approach consisting on adapting WSAN nodes transmission power to assure each node has a minimum number of neighbors, as described previously. Vicente Hernández Díaz, Néstor Lucas Martínez and Raúl M. del Toro carried out and deployed in the SunSPOT nodes comprising the WSAN under study, the software modules and components described in previous sections. They also run the experiments and made the assesments taken into account the outcomes from the tests.

\section{Conflicts of Interest}

The authors declare no conflict of interest.

\section{References}

1. Petersen, S.; Aakvaag, N. Wireless Instrumentation for Safety Critical Systems. Technology, Standards, Solutions and Future Trends. Technical report, SINTEF ICT, Communication Systems, 2015.

2. Kotian, R.; Exarchakos, G.; Liotta, A. Assessment of proactive transmission power control for wireless sensor networks. Proceedings of the 9th International Conference on Body Area Networks. ICST (Institute for Computer Sciences, Social-Informatics and Telecommunications Engineering), 2014, pp. 253-259.

3. Mahmood, M.A.; Seah, W.K.; Welch, I. Reliability in wireless sensor networks: A survey and challenges ahead. Computer Networks 2015, 79, 166 - 187.

4. Kusy, B.; Richter, C.; Hu, W.; Afanasyev, M.; Jurdak, R.; Brunig, M.; Abbott, D.; Huynh, C.; Ostry, D. Radio diversity for reliable communication in WSNs. Information Processing in Sensor Networks (IPSN), 2011 10th International Conference on, 2011, pp. 270-281.

5. Huang, Y.; del Toro, R.M.; Martínez Ortega, J.F.; Hernández Díaz, V.; Haber, R. Connectivity control in WSN based on fuzzy logic control. ACM SIGBED Review - Special Issue on the 6th Workshop on Adaptive and Reconfigurable October 2014, 11, 54-57.

6. Díaz, V.H.; Martínez, J.F.; Martínez, N.L.; del Toro, R.M. Self-Adaptive Strategy Based on Fuzzy Control Systems for Improving Performance in Wireless Sensors Networks. Sensors 2015, $15,24125$.

7. Zhang, H.; Hou, J.C. Maintaining sensing coverage and connectivity in large sensor networks. Ad Hoc \& Sensor Wireless Networks 2005, 1, 89-124.

(C) 2015 by the authors; licensee MDPI, Basel, Switzerland. This article is an open access article distributed under the terms and conditions of the Creative Commons Attribution license (http://creativecommons.org/licenses/by/4.0/). 